\title{
COMPARATIVE EVALUATION OF GINGIVAL DEPIGMENTATION USING SCALPEL/ ELECTROCAUTERY/ DIAMOND BUR/ DIODE LASER. A CASE REPORT.
}

\section{Dr. Agarwal Priyanka* \\ Dr. Mudda Jayashree \\ Dr. Patil Veena}

\section{Dr. Desai Shrikar}

\section{Dr. Ansari T. Sobia}

\section{Dr. Sultana Shahnaaz}

Department of Periodontics and Implantology, H.K.E.S' S. Nijalingappa's Institute of Dental Sciences and Research, Kalaburagi, Karnataka. ${ }^{*}$ Corresponding Author

Department of Periodontics and Implantology, H.K.E.S' S. Nijalingappa's Institute of Dental Sciences and Research, Kalaburagi, Karnataka.

Department of Periodontics and Implantology, H.K.E.S' S. Nijalingappa's Institute of Dental Sciences and Research, Kalaburagi, Karnataka.

Department of Periodontics and Implantology, H.K.E.S' S. Nijalingappa's Institute of Dental Sciences and Research, Kalaburagi, Karnataka.

Department of Periodontics and Implantology, H.K.E.S' S. Nijalingappa's Institute of Dental Sciences and Research, Kalaburagi, Karnataka.

Department of Periodontics and Implantology, H.K.E.S' S. Nijalingappa's Institute of Dental Sciences and Research, Kalaburagi, Karnataka.

\section{ABSTRACT Introduction: Gingival pigmentation which is caused by deposition of melanin pigment is a major} esthetic concern for many people. A case is reported in which an evaluation of 4 popular depigmentation

procedures carried.

Methodology: Patients associated with gingival hyperpigmentation in 4 quadrants based on Dummetts scoring criteria were included. Healing and recurrence were assessed following depigmentation carried out by scalpel, bur, electrosurgery and laser. The outcome of procedures were assessed at 1 week, 1 month and 3 months.

Results: Healing following Scalpel and laser were better than bur and electro surgery. Post-operative pain was less with the use of scalpel and laser.

Discussion: In this case report, it was found that the time taken to carry out all the 4 procedures were nearly the same. Both scalpel surgery and laser led to uneventful healing and minimal post-operative pain when compared to abrasion by burs and electro surgery in the immediate post-surgical period of 1 week to $l$ month.

Conclusions: Better treatment outcomes were observed with the scalpel and laser techniques.

\section{KEYWORDS : Depigmentation, scalpel, electrocautery, diamond bur, diode laser.}

\section{INTRODUCTION}

Melanin hyperpigmentation of gingiva in oral cavity usually present an issue of aesthetic origin especially in the anterior labial gingiva and is combined with display of gingiva while smiling. ${ }^{1}$ The methods which have been implemented over the years for gingival depigmentation include gingivectomy with free gingival autografting, cryosurgery, electrosurgery, chemical agents such as 90\% phenol and 95\% alcohol, acellular dermal matrix allograft, abrasion with diamond bur, Nd: YAG laser, semiconductor diode laser, CO2 laser has been used for cosmetic therapy for gingival depigmentation. ${ }^{2}$

\section{CASE REPORT}

18 year old male patient visited to the Department of periodontics with the chief complaints of black colored gums since childhood visible in upper and lower front region of jaw while smiling. There was no remarkable medical history or any oral destructive habit. On examination diffused melanin hyperpigmentation was found on the labial surface of both the maxillary and mandibular arches. Dummett: Gupta Oral Pigmentation Index score was 3.(Figure 1) Considering the patient's concern, surgical depigmentation procedure was planned. The entire procedure was explained to the patient and written consent was obtained. Routine oral hygiene procedures were carried out and oral hygiene instructions were given. To rule out any contraindication for surgery, a complete blood investigation were done.

Local anaesthesia was infiltrated in the maxillary and mandibular anterior region from first premolar to first premolar.
A conventional scalpel technique was used, where a BardParker handle with a \#15 blade was used to remove the pigmented layer from 31 to 34 with scrapping technique. Pressure was applied with sterile gauze soaked in local anaesthetic agent to control haemorrhage during the procedure. After removing the entire pigmented epithelium along with a thin layer of connective tissue with scalpel, care was taken to see that all remnants of the pigmented layer was removed.

Maxillary anterior gingiva from 21 to 24 depigmentation procedure was performed with electrocautery; a loop electrode was used for deepithelizing the gingiva. It is used in a light brushing strokes and tip is kept in motion. Keeping tip in one place could lead to excessive heat build-up and leads to destruction of tissues.

With laser technique, depigmentation procedure, was performed in mandibular anterior gingiva from 41 to 44 by diode laser with Sieve method. A fibreoptic laser tip kept in contact with the pigmented area and laser was emitted in a gated pulsed mode and operated with the wavelengths of 980 $\mathrm{nm}$. Radiation points were as close as possible to each other, although the fiber was moved enough not to overlap the previous site. Depigmentation was performed with in a horizontal direction to remove the epithelial lining. The area to be depigmented was wiped with gauze soaked in saline solution and the same procedure was repeated till no pigments remained.

A diamond bur was used for depigmentation from 14 to 11 . Revolving bur was moved with feather light strokes without 
giving any pressure. It was not kept at one place for long time as it may result in thermal trauma and permanent harm to underlying tissue. The bleeding was controlled and checked for any pigmented area if remained and was removed to prevent relapse.

Finally, after completing the procedure the surgical area (Figure 2) was covered with a periodontal dressing pack to prevent foreign irritants, thermal stimuli, and infection. Postsurgical instructions were given to patient and was advised antibiotics (Amoxicillin 500mg, thrice daily for five days), analgesics (Ibuprofen with paracetamol, thrice daily for three days) and chlorhexidine mouthwash (12 hourly for one week).The outcome of all the procedures were assessed at 1 week, 1 month and 3 months for healing and recurrence of pigmentation. (Figure3)

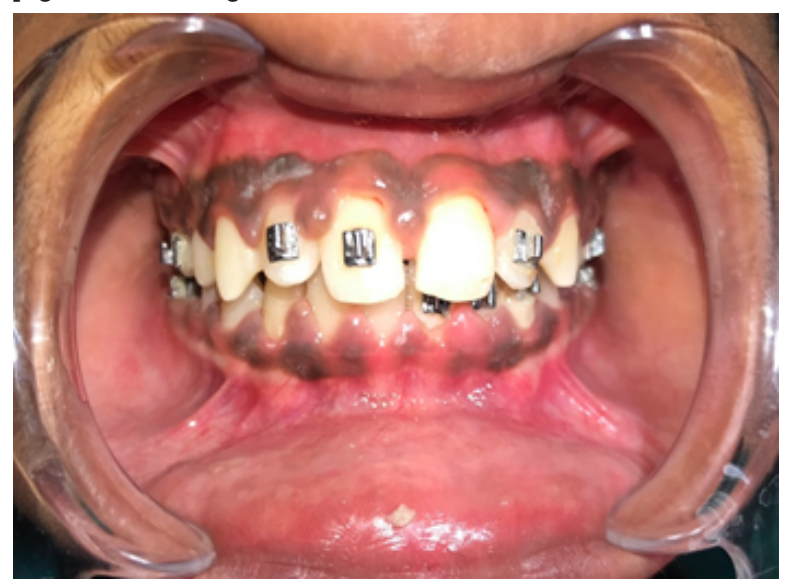

Figure 1

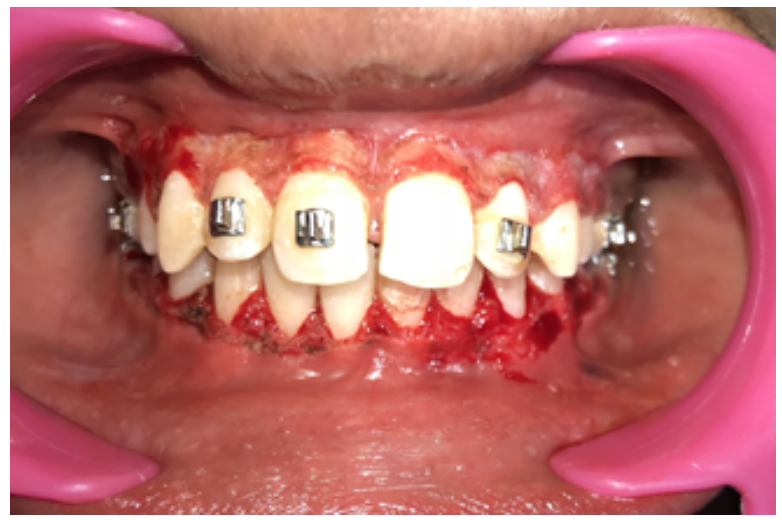

Figure 2

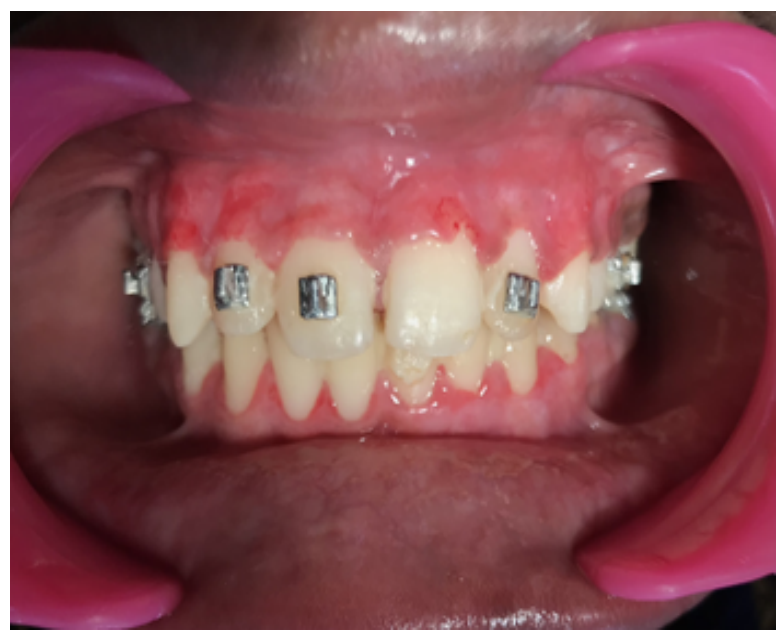

Figure 3

\section{RESULTS}

At the $1^{\text {st }}$ week of follow up all four procedure were assessed. Healing was satisfactory in the sites treated with scalpel and laser. In addition healing appeared to be delayed with persistent granulation tissue and increased redness of the gingival tissue in the sites treated by the bur technique; and delayed with the formation of a scar in the electrosurgery treated sites, which persisted until 1 month following the procedure. However at the end of 2 months and thereon, the pain had completely subsided and healing was complete. And no recurrence of pigmentation was seen with all the four procedure at the end of 3 months.

\section{DISCUSSION}

Gingival health and appearance are the essential components of an attractive smile. Gingival depigmentation is a periodontal plastic surgical procedure whereby the gingival hyperpigmentation is removed or reduced. The first and foremost indication for depigmentation is patient demand for improved esthetics. The intensity and distribution of pigmentation of the oral mucosa is variable ,not only between races but also between different individuals of the same race and within different areas of the same mouth. Melanin pigmentation is frequently caused by melanin deposition by active melanocytes located mainly in the basal layer of the oral epithelium. The selection of a technique for depigmentation of the gingiva should be based on clinical experience, patient's affordability and individual preferences.

This case report was an attempt to evaluate various techniques of gingival depigmentation- viz scalpel surgery, abrasion by a diamond bur, electro surgery and laser. All the four techniques were carried out in the same patient in a split mouth design so as to avoid bias related to the host with regard to healing and recurrence.

In this case report, it was found that the time taken to carry out all the 4 procedures were nearly the same. In addition, it was also observed that both scalpel surgery and laser led to uneventful healing and minimal post-operative pain when compared to abrasion by burs and electro surgery in the immediate post-surgical period of $l$ week to $l$ month. However, at the end of 3 months follow-up period all 4 techniques were comparable to each other.

Scalpel surgical technique is highly recommended as it is simple, easy to perform, cost effective and above all causes minimum discomfort and is also esthetically acceptable to patient. The healing period for scalpel wounds is faster than other techniques. However, scalpel surgery may cause unpleasant bleeding during and after the procedure. According to Almas and Sadiq (2002), ${ }^{3}$ the scalpel wound heals faster than that in other techniques. In our study also, the healing by scalpel was faster than that by electrocautery and diamond bur. But scalpel surgery causes unpleasant bleeding during and after the operation. It is also necessary to cover the exposed lamina propria with periodontal dressing for 7-10 days. ${ }^{4}$

Diamond bur techniques do not require any sophisticated equipment and are hence economical. Pre- and post-surgical care is similar to that of the scalpel technique. However, extra care should be taken to control the speed and pressure of the bur so as not to cause unwanted abrasion or pitting of the tissue. Feather light brushing strokes with minimum pressure and copious saline irrigation should be used without holding the bur in one place to get excellent results. ${ }^{5}$

Electro-surgery requires more expertise than scalpel surgery. This technique is uncomfortable to patients due to foul odour and the use of high-speed suction is mandatory. Electrosurgery has a strong influence in retarding migration of melanin cells 
from the locally situated cells ${ }^{6}$ but also has its own limitations in that, its repeated and prolonged use induces heat accumulation and undesired tissue destruction. Cicek (2003) ${ }^{7}$ reported that there is no bleeding and there is minimal patient discomfort while using electrocautery. But electrosurgery also has its own limitations in that its repeated and prolonged use induce heat accumulation and undesired tissue destruction.

Another effective treatment modality employed in the present case is by diode laser. Here, radiation energy is transformed into ablation energy, resulting in cellular rupture and vaporization with minimal heating of the surrounding tissue. According to Atsawasuwan and Greethong (1999), ${ }^{8}$ laser beam produces bloodless field for surgery, causes minimum damage to the periosteum and underlying bone. This has the advantages of easy handling, short treatment time hemostasis, and decontamination and sterilization effects. But this approach needs expensive and sophisticated equipment, which makes the treatment very expensive.

A recent case series comparing gingival depigmentation procedures by 3 techniques- scalpel surgery, abrasion by a diamond bur and electrosurgery found that the scalpel surgical technique gave results as good as the other techniques. The post-operative complications and scar formation was lesser than with the electrosurgical procedure. However, the treatment time required was high compared to the other techniques. ${ }^{9}$

Interestingly, no recurrence of pigmentation was observed in all 4 procedures at the end of 3 months. This is a very important aspect of depigmentation treatment outcomes as reemergence of pigmentation is a common occurrence.

\section{CONCLUSION}

Cosmetic expectations have increased with time and nowadays patients are more concerned with gingival esthetics and smile designing. Gingival pigmentation especially on the labial aspect of anterior teeth has become an important component of esthetics. Within the limitations of the present study, depigmentation by scalpel and laser have shown better results than bur and electrocautery techniques. Although the scalpel was most economical, with regard to healing and recurrence of pigmentation, all four techniques were at par with each other. Each technique has its own advantages and disadvantages. By using diode lasers, it has been documented that there is minimal postoperative bleeding, pain, wound healing, discomfort, and ease of the procedure. However, drawback of this study is shorter followup period, and there is no histological and histochemical assessment for the activity of melanocytes.

\section{REFERENCES}

1. A Karydis, Bland P, Shiloah J. "Management of Oral Melanin Pigmentation" Journal of the Tennessee Dental Association 2012 ; 92 : 10-16.

2. Fowler EB, Breault LG, Galvin BG. Enhancing physiologic pigmentation utilizing a free gingival graft. Pract Periodontics Aesthet Dent 2000;12:193-96.

3. Almas K, Sadiq W. Surgical Treatment of Melanin-Pigmented Gingiva: An Esthetic Approach. Indian J Dent Res 2002; 13:70-3.

4. Pal TK, Kapoor KK, Parel CC, Mukharjee K. Gingival melanin pigmentation: A study on its removal for esthetics. J Indian Soc Periodontol 1994;3:52-4.

5. Putter OH, Ouellet D, Putter A, Vilaboa D, Vilaboa B, Fernandez M. A nontraumatic technique for removing melanotic pigmentation lesions from the gingiva: Gingiabrasion. Dent Today 1994; 13: 58-60.

6. Hegde R, Padhye A, Sumanth S, Jain AS, Thukral N. Comparison of Surgical Stripping, Er: YAG Laser and CO Laser Techniques for Gingival 2 Depigmentation: A Clinical \& Histological Study. J Periodontol 2013;84(6):72848. doi:1902/jop.2012.120094.

7. Y. Cicek, "The normal and pathological pigmentation of oral mucous membrane: Areview,"Journal of Contemporary Dental Practice 2003; 4(3), 76-86.

8. Atsawasuwan P, Greethong K, Nimmanon V. Treatment of gingival hyperpigmentation for esthetic purposes by Nd: YAG laser: Report of 4 cases. J Periodontol 2000;71:315-21

9. S. K. Kasagani, R. Nutalapati, and R. B. Mutthineni, "Esthetic depigmentation of anterior gingiva-A case series," New York State Dental Journal, vol. 78, no.3, pp. 26-31, 2012. 